\title{
Computer-Aided Evaluation Protective Zone of Transmission Lines Using Remote Sensing Multispectral Data
}

\author{
Olga V. Grigorieva, Andrey V. Markov, Alisher G. Saidov, Elena I. Astahova \\ A.F. Mozhaisky Military Space Academy, Saint-Petersburg, Russia \\ alenka12003@mail.ru
}

\begin{abstract}
This work presents the original software to monitor compliance with the established conditions of the protective zone of transmission lines. Remote sensing data in the visible and infrared spectral ranges are used as input. The software provides the computer-aided creation of thematic digital layers using remote sensing multispectral data. The layers contain spatial and attributive information about violations affecting the safe operation of electric grid facilities. There is a brief description of the methods and algorithms developed to solve the problems of estimation of wood overgrowth along transmission lines and identification of typical objects (landfill, oil-contaminated sediment, soil damage) that are prohibited in the protective zone.
\end{abstract}

Keywords: protective zone of transmission lines, evaluation of plant occurrence, monitoring of land-use violation, remote sensing multispectral data processing, software 


\title{
АВТОМАТИЗИРОВАННОЕ ОЦЕНИВАНИЕ СОСТОЯНИЯ ОХРАННЫХ ЗОН ЛИНИЙ ЭЛЕКТРОПЕРЕДАЧ ПО ДАННЫМ КОСМИЧЕСКОЙ МНОГОСПЕКТРАЛЬНОЙ СЪЕМКИ
}

\author{
О.В. Григорьева, А.В. Марков, А.Г. Саидов, Е.И. Астахова \\ Военно-космическая академия имени А.Ф. Можайского, Санкт-Петербург, Россия \\ alenka12003@mail.ru
}

В статье рассматривается оригинальное программное обеспечение, позволяющее на основе анализа материалов дистанционного зондирования Земли в видимом и ближнем инфракрасном диапазонах спектра оценивать соблюдение условий использования земельных участков в пределах охранной зоны воздушных линий электропередач. Реализованный в программе методический аппарат обработки данных космической многоспектральной съемки обеспечивает создание в автоматизированном режиме тематических слоев электронных карт, которые содержат пространственную и атрибутивную информацию о характерных нарушениях, влияющих на безопасное функционирование и эксплуатацию объектов электросетевого хозяйства. Приводится краткое описание методов и алгоритмов, разработанных для решения задач оценивания степени зарастания древесной растительностью просек вдоль линий электропередач и выявления типовых объектов (свалок, участков нефтезагрязнений и механических повреждений почв), запрещенных в пределах охранной зоны.

Ключевые слова: охранная зона воздушных линий электропередач, оценивание степени зарастания растительностью, контроль нарушений землепользования, тематическая обработка данных космической многоспектральной съемки, программное обеспечение.

\section{Введение}

Контроль соблюдения особых условий использования земельных участков, расположенных в пределах охранных зон воздушных высоковольтных линий электропередач (ЛЭП), имеет важное значение для обеспечения безопасных условий эксплуатации и исключения возможности повреждения этих объектов электросетевого хозяйства. Одним из наиболее эффективных способов решения данной задачи, ввиду значительной протяженности ЛЭП, является проведение периодической авиационной или космической съемки охранных зон в сочетании с последующим наземным дообследованием выявленных проблемных участков. При этом большие объемы регистрируемых данных определяют актуальность автоматизации процесса их дешифрирования.

В настоящей работе приведено краткое описание оригинального программного обеспечения, предназначенного для автоматизированной обработки материалов космической съемки в интересах оценивания состояния охранных зон ЛЭП на предмет установления степени их зарастания и выявления фактов (свалок, нефтезагрязнений и механических повреждений почв), свидетельствующих о нарушениях условий землепользования. 


\section{Программное обеспечение автоматизированного оценивания состояния охранных зон воздушных ЛЭП}

При проведении аэрокосмического мониторинга охранных зон воздушных ЛЭП, как правило, решаются задачи оценивания степени зарастания просек вдоль ЛЭП древесной растительностью и выявления объектов, запрещенных к размещению на контролируемой территории. При этом преимущественно задействуется оптико-электронная съемочная аппаратура, регистрирующая панхроматические (ПХ) или цветосинтезированные (ЦС) изображения. Анализ полученных снимков производится методом визуального дешифрирования, к недостаткам которого относятся низкая оперативность и зависимость результатов от квалификации оператора.

Вместе с тем современные космические системы дистанционного зондирования Земли (Д33) способны предоставить данные многоспектральной съемки (МСС), при обработке которых целый ряд задач оценивания состояния охранных зон ЛЭП возможно решить в автоматическом или автоматизированном режиме. В частности, известны методические подходы [1-6] позволяющие использовать данные МСС в интересах следующих тематических приложений:

- оценивания степени зарастания просек вдоль ЛЭП (с целью предотвращения возможных разрывов проводов и контроля обеспечения доступа к ЛЭП) на основе определения характеристик деревьев, находящихся в охранной зоне;

- выявления запрещенных к размещению в охранных зонах свалок;

- обнаружения загрязнений территории нефтепродуктами, свидетельствующих о фактах сброса (слива) горюче-смазочных материалов;

- обнаружения механических повреждений почв (фактов земляных работ, сельскохозяйственных работ, связанных с вспашкой земли, и т.п.), которые могут привести к повреждению опор ЛЭП.

Для практической реализации методического аппарата решения перечисленных тематических задач было разработано специальное программное обеспечение, структурная схема которого представлена на рисунке 1. В состав программы входит пять программноалгоритмических модулей автоматизированной и интерактивной тематической обработки данных Д33, а также база данных (БД) эталонных изображений и дешифровочных признаков (ДП) типовых объектов, запрещенных к размещению в охранной зоне.

В результате применения модулей автоматизированной обработки данных МСС в пределах границ обозначенной на снимках охранной зоны (импортируются из сторонних информационных ресурсов или определяются автоматически относительно ЛЭП в соответствии с имеющимися нормативами) формируются тематические слои электронной карты в формате SHP, в которых содержатся рассчитанные полигоны участков древесной растительности и выявленных нарушений землепользования.

Исходя из анализа пространственных и спектральных характеристик рассматриваемых объектов интереса [7] для получения результатов автоматизированной обработки требуемого качества необходимо использовать данные МCC, имеющие линейное разрешение на местности не хуже 3 м, в следующих интервалах спектра: 480-520 нм (синий); 540-600 нм (зеленый); 620-680 нм (красный 1); 660-690 нм (красный 2), 700-740 нм (крайний красный), 720-800 нм (БИК1) и 800-900 нм (БИК2). При этом материалы съемки должны иметь географическую привязку и быть обеспечены радиометрической калибровкой (с учетом атмосферной коррекции).

Интерактивный режим предусмотрен для предоставления оператору возможности уточнения (редактирования) сформированных тематических слоев электронной карты. Данная операция является актуальной при наличии на контролируемую территорию высокодетальных ПХ или ЦС снимков, при дешифрировании которых могут быть обнаружены дополнительные нарушения, не выявляемые в автоматическом режиме. Для информационной поддержки работы оператора в интерактивном режиме используется специализированный тематический цифровой классификатор условных знаков. 


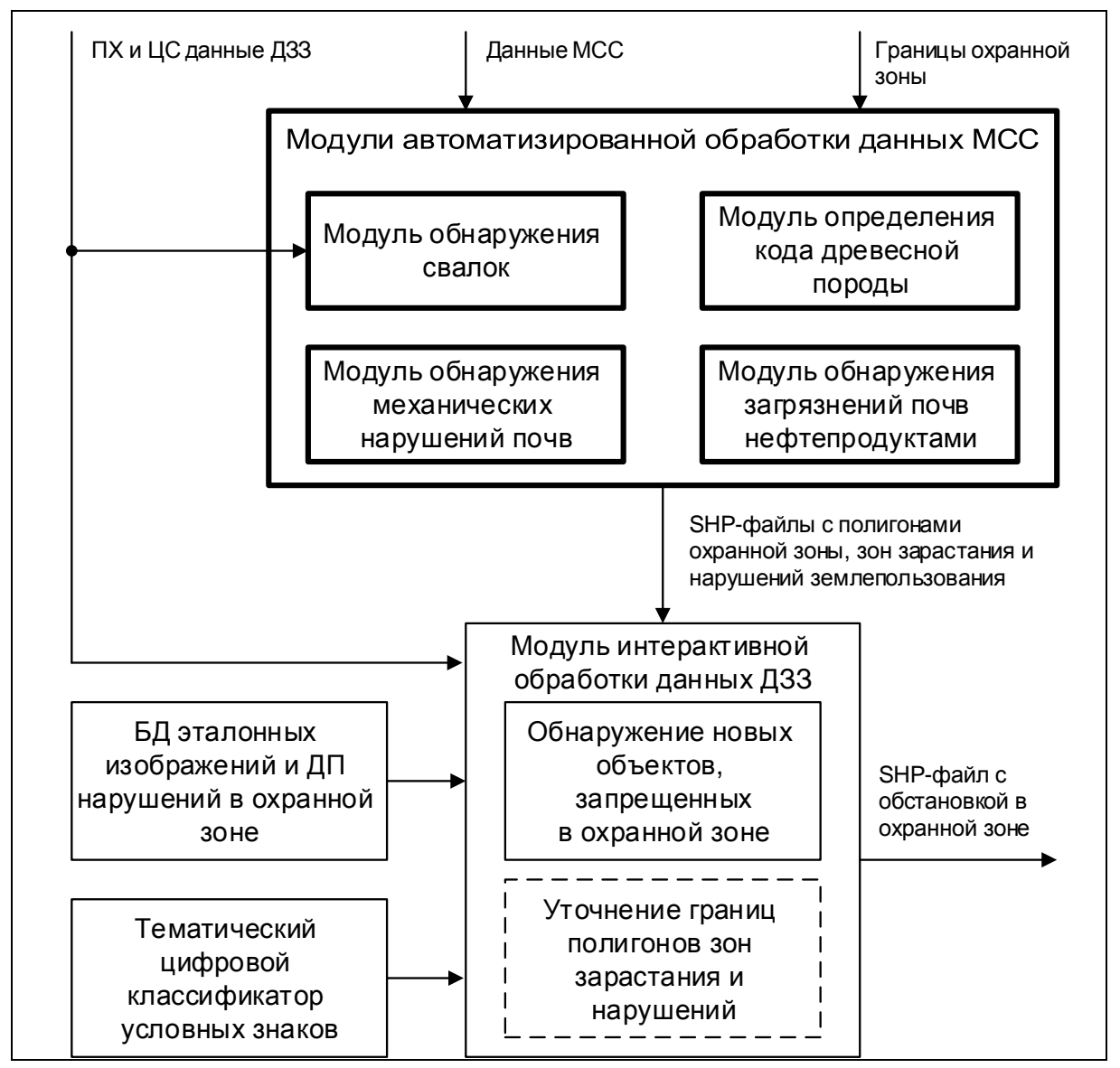

Рис. 1. Структура программного обеспечения автоматизированного оценивания состояния охранной зоны ЛЭП по данным ДЗЗ

\section{Методы и алгоритмы автоматизированной обработки данных МСС в интересах оценивания состояния охранной зоны ЛЭП}

Рассмотрим кратко методы и алгоритмы автоматизированной обработки данных МСС, реализованные в представленном программном обеспечении.

В задаче определения кода древесной породы первоначально производится разделение лесных и нелесных участков с созданием маски леса с помощью алгоритма релаксационной разметки, где в качестве признака используется вегетационный индекс NDVI. Это позволяет исключить влияние достаточно высоких значений метрических показателей в пространстве признаков при дальнейшей классификации оставшихся фрагментов.

Дальнейшая классификация породного состава предусматривает использование встроенной БД спектральных характеристик основных лесообразующих пород и их статистических оценок (коэффициентов вариации или среднеквадратических отклонений). Каждая спектральная сигнатура в БД сопровождается информацией о проективном покрытии (сомкнутости) (за исключением отдельных крон и элементов дерева); фенологическом периоде наблюдения (предусмотрено 8 фенофаз); преобладающей породе или составе древостоя для смешанных лесов. Дополнительно учитывается тип леса, характеризующийся общностью лесорастительных условий (комплексом климатических, гидрологических и почвенных факторов), и бонитет (показатель роста и потенциальной производительности насаждений для данных условий местопроизрастания).

Алгоритм обработки заключается в последовательном использовании метода классификации с построением обучающей выборки по результатам кластеризации. В качестве методов кластеризации в зависимости от типа данных применяются методы на подобии К- 
средних или нечеткой кластеризации, а в качестве методов классификации - методы максимального правдоподобия или нейронных сетей. Обучающие выборки назначаются по кластерам, для этого оценивается компактность кластера путем анализа индексов внутриклассового расстояния и количества локальных максимумов функции плотности распределения, описываемой с помощью многомерной оценки Парзена-Розенблатта. При этом объем выборки определяется пространственным разрешением данных и количеством спектральных каналов, участвующих в классификации.

Установление соответствия между пикселями класса и породным составом осуществляется с использованием специальных критериев принадлежности, выраженных в виде вероятности правильного отождествления спектров, специальных метрик (Махаланобиса и Терибижа) и коэффициента корреляции.

Каждой установленной в результате проведенной классификации древесной породе присваивается код (условное буквенное обозначение, например, Б - береза). В атрибутивные данные электронного слоя записывается формула породного состава, в которой приводятся коды древесных пород и доля участия каждой древесной породы, выражаемые в виде коэффициентов (целых чисел), каждая единица которых соответствует 10 процентам участия древесной породы в общем запасе древесины выдела. Например, для лесного насаждения, в котором присутствует 40 \% сосны и 60 \% березы, береза признается преобладающей породой и формула состава имеет вид 4С6Б.

Пример работы программного модуля, реализующего описанный алгоритм, приведен на рисунке 2.

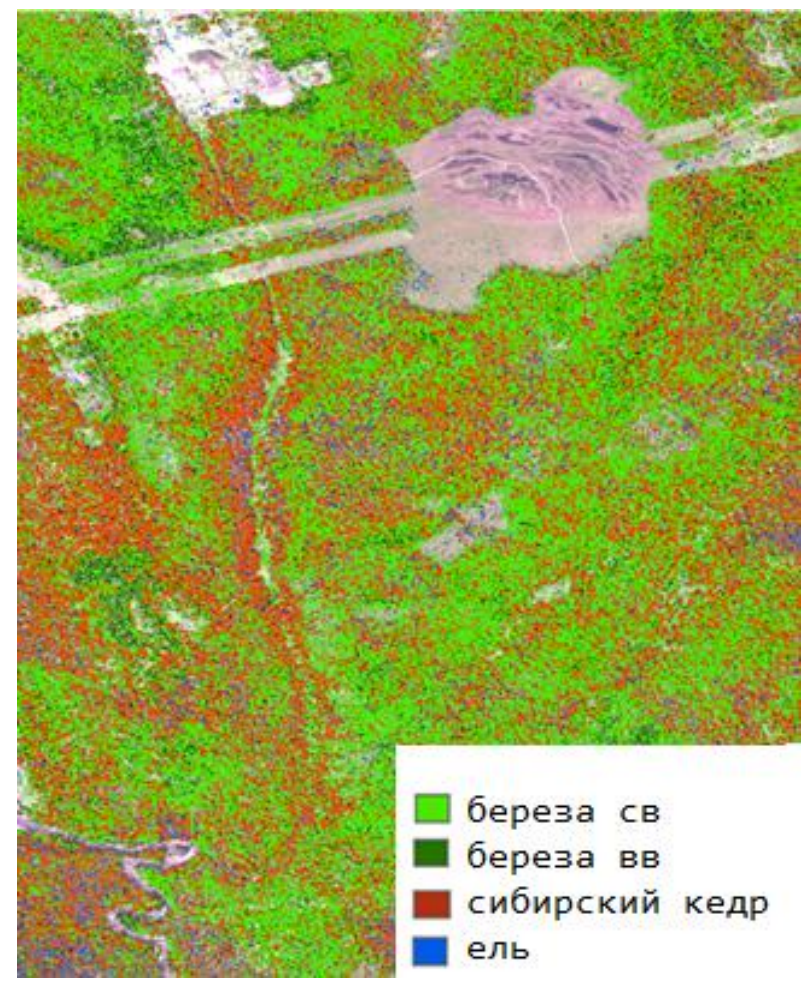

Рис. 2. Определение породного состава леса по данным МСС

Для обнаружения мест механического повреждения почв применяется алгоритм обработки данных МСС (в синем, зеленом, красном 2 и БИК1 каналах), основанный на кластеризации изображения методом нечетких множеств и последующем распознавании объектов интереса по их информативным (характерным для почвогрунтов) спектральным признакам.

В модуле обнаружения загрязнений почв нефтепродуктами (НП) алгоритм обработки данных МСС предусматривает выполнение следующих операций: 
1) Проводится неконтролируемая кластеризация трех индексных изображений NDVI методом нечетких множеств, где в качестве нормы разделения кластеров используется расстояние Евклида, разбивающая анализируемую область на три кластера. Индексные изображения NDVI рассчитываются в трех спектральных интервалах: крайнем красном, БИК1 и БИК2.

2) Из зоны дальнейшего поиска по пороговым значениям вегетационного индекса NDVI исключаются кластеры, соответствующие растительному покрову.

3) Для оставшихся кластеров проводится повторная кластеризация методом Isodata по всем исходным данным МСС и выделение кластеров, соответствующих по своим спектральным признакам нефтезагрязненным почвам. В качестве критериев отнесения кластера к участку нефтезагрязнения используются следующие меры сходимости спектральных сигнатур: расстояние Евклида, учитывающее разницу средних значений коэффициента яркости кластеров в спектральном канале, и коэффициент корреляции эталонных спектров НП и анализируемых спектров кластеров.

4) Осуществляется идентификация выделенных участков нефтезагрязнения по типу НП (битумизированный кир, жидкая фаза НП, нефтяная пленка и водонефтяная эмульсия) и степени загрязнения почв (полное разрушение, высокий, средний, низкий уровень) с использованием эталонных спектральных признаков почв и НП. При этом уровень загрязнения почв определяется по индексу нефтезагрязнености IS, рассчитываемому в БИК2 и синем спектральных каналах.

Алгоритм обнаружения свалок основан на анализе их спектральных и текстурных характеристик и включает в себя следующие процедуры:

- кластеризацию изображения с помощью метода нечетких множеств;

- обнаружение кластеров свалок по спектральным признакам отходов различного морфологического состава;

- распознавание кластеров свалок по текстурным и частотно-контрастным признакам (в данной процедуре наряду с данными МСС могут эффективно использоваться также ПХ или ЦС изображения).

По результатам выполнения перечисленных процедур в качестве выходных данных формируется файл формата SHP, отображающий контуры выявленных свалок (рисунок 3), в котором выделенные полигоны обеспечены атрибутивной информацией о морфологическом составе, площади, классе опасности свалки и степени ее негативного воздействия на окружающую среду.

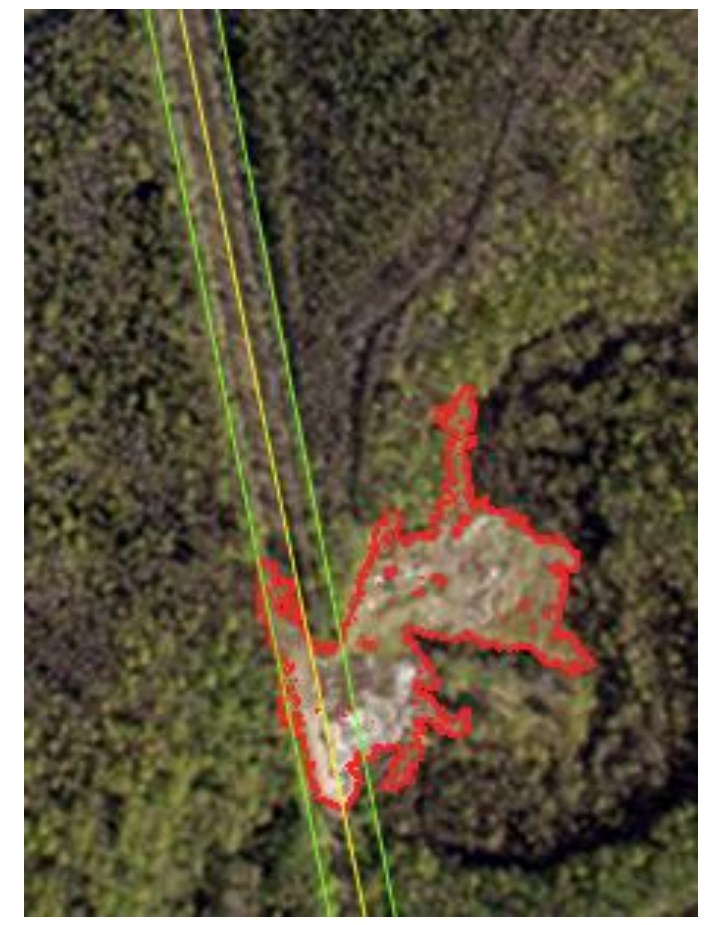

Рис. 3. Обнаружение несанкционированной свалки в пределах охранной зоны ЛЭП 


\section{References}

[1] Grigorieva O.V., Saidov A.G., Panin A.V. The status indicator of the soils in the areas of oil, obtained by non-contact means of observations, Ecology and industry of Russia, 2010, October, pp.5053. (In Russian).

[2] Brovkina O.V., Skoropisov D.Y. Remote sensed monitoring of dumps (in Kronshtadt district of Saint-Petersburg), Modern problems of remote sensing of the Earth from space, 2012, Vol. 9, No 1, pp. 153-155. (In Russian).

[3] Grigorieva O.V. , Zhukov D.V. , Markov A.V. The development of efficient algorithms for thematic processing of hyperspectral systems for the identification of landscape objects, Scientific and technical journal «Contenant», 2013, Vol.12, No 2., pp. 90-99. (In Russian).

[4] Makarov A., Grigorieva O., Brovkina O., Mochalov V., Zhukov D. Automated methods for environmental assessment based on multi- and hyperspectral imagery, Geomatics, 2012, No 4, pp.102106.

[5] Markov A., Grigoryeva O., Saidov A., Mochalov V., Zhukov D. The set of software tools for thematic processing of hyper- and multispectral satellite, Geomatics, 2015, No 1, pp.32-37.

[6] Grigorieva O., Brovkina O., Mochalov V., Akhtman Y., Zelentsov V., Potryasaev S., Kozyr I., Belova N. A complex model for forest ecosystem state assessment based on remote sensing data: case study in Baikalsky Nature Reserve. In The 4th International Workshop on Simulation for Energy, Sustainable Development and Environment, Cyprus, 2016. pp. 14-19.

[7] Markov A., Grigoryeva O., Saidov A., Methods of a substantiations of requirements to parameters of multispectral optical-electronic means of remote sensing. In V International Scientific and Technical Conference Actual problems of Creation of Space Systems of Earth Remote Sensing, VNIIEM Corporation JC, Moscow, Russian, 2017. pp. 65-67. 